\title{
ERSCHEINUNGSFORMEN DES MEHRWERTS VON PHRASEOLOGISMEN IN DEUTSCHEN WERBETEXTEN
}

\author{
Anna-Mariia Mashchenko \\ Doktorandin des Lehrstuhls für germanische und finnisch-ungarische Philologie \\ namens Professor H. H. Pocheptsov, Nationalen linguistischen Universität Kyiv, Ukraine \\ e-mail: arm-ann@ua.fm, orcid.org/0000-0002-2362-3433
}

\section{Zusammenfassung}

Der Artikel ist der Untersuchung des semantischen Mehrwerts der Phraseologismen, den Besonderheiten seines Ausdrucks in den festen Wortkomplexen verschiedener Art gewidmet, die den Werbetexten deutschsprachiger Zeitschriften "Der Spiegel", "Psychologie Heute", "Schöner wohnen" und "Brigitte" entnommen wurden. Als Ergebnis der Analyse von 654 stehenden Redewendungen, die in 313 Werbetexten enthalten sind, wurden drei Gruppen von Phraseologismen entsprechend den Besonderheiten ihrer phraseologischen Bedeutung ausgesondert, die konnotativ gewissermaßen besonders geladen sind und sich nach der Meinung der deutschen Linguisten durch einen semantischen Mehrwert auszeichnen, den die ukrainischen Sprachwissenschaftler einen besonderen Zusatz nennen. Am deutlichsten kommt der Mehrwert bei den Phraseologismen mit idiomatischer, d. h. völlig umgedeuteter Bedeutung, etwas schwächer - bei den phraseologischen Einheiten mit idiophraseomatischer, $d$. h. teilweise umgedeuteter Bedeutung zum Ausdruck. Vom Mehrwert der stehenden Redewendungen mit phraseomatischer, d. h. verstärkter, aber nicht umgedeuteter Bedeutung, zeugen ihre Verbundenheit mit Schriftsprache und Funktionalstil sowie ihre Eigenschaft, das sinnwichtige Wort durch verstärkte Betonung auszusondern. Im Artikel werden die wichtigsten Möglichkeiten des Ausdrucks vom Mehrwert der Phraseologismen im Werbetext analysiert: übertragene Bedeutung der Redewendungen, Ungewöhnlichkeit der Situation, in die sie vom Autor eingebettet werden, unerwarteter Gebrauch der Phraseologismen in ihrer freien Bedeutung, Einsatz von Sprichwörtern, Zitaten aus bekannten literarischen Werken, Kinderreimen, geflügelten Worten, Kommentieren des Phraseologismus des Titels im Text der Werbung u. Ä. Besonders beeindrucken den Rezipienten modifizierte phraseologische Einheiten verschiedenen Charakters: Erweiterung und Reduktion des Ausdrucks, Substitution, Kontamination sowie Veränderung der grammatischen Struktur. Ein spezifisches Mittel der deutschen Sprache ist die Erweiterung des Ausdrucks durch Bildung von Zusammensetzungen. Die lexikalische Ausfüllung der Werbetexte zeugt von ständiger Aufmerksamkeit der Textautoren zum Rezipienten, von Berücksichtigung seiner Interessen und seines Niveaus.

Schlüsselwörter: phraseologische Bedeutung, phraseomatische Bedeutung, idiophraseomatische Bedeutung, idiomatische Bedeutung, Expressivität, Intensivität, Modifikation.

DOI: https://doi.org/10.23856/3809

\section{Einleitung}

Die aktuellen phraseologischen Untersuchungen sind eng mit Problemen der Semantik und des Funktionierens von emotionsgeladenen Spracheinheiten im Text verbunden (V. Shakhovskyi, 2008). Die vorliegende wissenschaftliche Abhandlung gehört auch zu Untersuchungen, die mit der Feststellung von Mechanismen des Ausdrucks von Emotionen mit Mitteln der Phraseologie zusammenhängen. Bei der Analyse der Wortbedeutung betrachten 
die heutigen Linguisten das Denotat als Entsprechung des Bezeichneten, das Signifikat als Begriffskern der Bedeutung und das Konnotat als Zusatzkomponente, die gegenständlich-logischen Gehalt der lexikalischen Einheit durch ein Bündel der subjektiven Merkmale von Emotionalität, Expressivität, funktional-stilistischer Färbung ergänzt, die durch Merkmale von sozialen, ideologischen, kulturellen und situativen Aspekten der Kommunikation bedingt sind (O. Selivanova, 2010: 114, 281, 645).

Die heutigen Linguisten vertreten die Meinung, dass Phraseologismen und Wörter viele gemeinsame Züge haben, dass ihre Bedeutungen miteinander korrelieren, was ihnen die Möglichkeit gibt, syntaktische Verbindungen miteinander einzugehen (Y. Baran, 2008: 82; L. Avksentiev, 1988: 23). Dabei fallen die semantischen Strukturen der Wörter und die semantischen Strukturen der Phraseologismen, die zu der gleichen lexikalisch-grammatischen Kategorie gehören, niemals völlig zusammen (A. Kunin, 1996:35). Der ukrainische Linguist V. Uzhchenko präzisiert diesen Gedanken, indem er darauf hinweist, dass zu einer phraseologischen Wendung noch etwas "über dem Wort", "mehr als das Wort", irgendein "Zusatz" gehört. Er meint, dass die Bedeutung eines Phraseologismus komplizierter ist als die eines Wortes, die "den Kern des einheitlichen Begriffs nur äußerst knapp wiedergibt" (V. Uzhchenko, 1988: 8). Der deutsche Sprachwissenschaftler P. Kühn vertritt die Meinung, dass Phraseologismen pragmatisch "besonders geladen" sind und sich durch einen semantischen Mehrwert auszeichnen. Sie treten im Satz als semantisch hochgradig komplexe, kompakte sprachliche Zeichen auf (P. Kühn, 1994: 420). Eine ähnliche Meinung äußert auch der deutsche Linguist H. Burger, der vom konnotativen Mehrwert der Phraseologismen spricht, indem er auf ihre Expressivität hinweist, die verschiedene Aspekte erfasst (H. Burger, 2010: 81). Von einem solchen Mehrwert der festen Wortkomplexe zeugt auch die Tatsache, dass ihr Ersatz durch nichtphraseologische Synonyme zum Verlust an Anschaulichkeit, Bildlichkeit und Expressivität führt. Den Phraseologismen ist ein "semantisches Element der Intensität" eigen (W. Fleischer, 1982: 221, 205). Beim Einsatz in der Werbung erscheint uns diese Eigenschaft der festen Wortkomplexe besonders wichtig. Die Verfasser der Werbetexte versuchen "auf engstem sprachlichem Raum dem Hörer bzw. Leser ein Produkt so umfassend und anschaulich wie möglich zu präsentieren; die positive Einstellung des Werbeproduzenten seiner Ware gegenüber soll mit der Hilfe von Phraseologismen sprachökonomisch gerafft auf den potentiellen Käufer übertragen werden” (D. Drumm, 2004: 109).

Im Hinblick darauf, dass der Werbetext auf eine bestimmte Weise den Rezipienten beeinflussen und überzeugen soll, halten wir es für wichtig, die möglichen Arten dieses Einflusses zu untersuchen, darunter auch durch die Erforschung der Einwirkung verschiedener Erscheinungsformen des Mehrwerts. Dementsprechend besteht das Ziel des Artikels im Herausfinden der Mittel und Möglichkeiten des Ausdrucks vom Mehrwert in festen Wortkomplexen verschiedener Typen, die in der deutschsprachigen Anzeigenwerbung vorkommen. Zur Verwirklichung dieses Ziels führt die Erfüllung von folgenden Aufgaben: die Aussonderung der einzelnen Gruppen von Phraseologismen des Untersuchungscorps und ihre Analyse auf die pragmatischen Besonderheiten.

Die Besonderheiten der Thematik erfordern den Einsatz verschiedener Forschungsmethoden. Unter den Methoden, die für unsere Untersuchung relevant erscheinen, sind vor allem die von A. Kunin vorgeschlagene phraseologische Identifikation und phraseologische Beschreibung zu nennen, die zur Aussonderung der einzelnen Forschungsgruppen von Phraseologismen und Feststellung ihrer Besonderheiten verhelfen. Die Methode der phraseologischen Analyse ermöglicht die Präzisierung von Details dieser Charakteristik. Von Bedeutung ist auch die Benutzung der von I. Arnold vorgeschlagenen Komponentenanalyse auf Grund der Wörterbuchdefinitionen. 


\section{Semantischer Mehrwert von Phraseologismen mit idiomatischer und idiophraseomatischer Bedeutung}

Im Rahmen der vorliegenden wissenschaftlichen Abhandlung wurden 654 stehende Redewendungen analysiert, die 313 Werbetexten aus den deutschsprachigen Zeitschriften "Der Spiegel", "Psychologie Heute", "Schöner wohnen" und "Brigitte" entnommen wurden. Die analysierten Phraseologismen wurden in drei Gruppen entsprechend den Besonderheiten ihrer phraseologischen Bedeutung geteilt: stehende Redewendungen mit phraseomatischer, d. h. verstärkter, aber nicht umgedeuteter Bedeutung (210 Einheiten), stehende Redewendungen mit idiophraseomatischer, $\mathrm{d}$. h. zum Teil mit verstärkter und zum Teil mit umgedeuteter Bedeutung (384 Einheiten) und stehende Redewendungen mit idiomatischer d. h. völlig umgedeuteter Bedeutung (60 Einheiten).

Am deutlichsten kommt der Mehrwert von Phraseologismen der letzten Gruppe zum Ausdruck, etwas schwächer - der vorletzten. Die Umdeutung dieser Phraseologismen kann durch den Vergleich mit ihren Prototypen mit dem gleichen lexikalischen Bestand oder mit der direkten Bedeutung der Komponenten aufgedeckt werden (A. Kunin, 1996: 142).

In der Werbung für den Film "Die Wunderübung" (Psychologie Heute, Februar 2019: 91) steht: Wir sehen, wie sich die Partner anzicken, wir fühlen mit und, ja, wir amüsieren uns über die Gemeinheiten, die sie sich an den Kopf werfen. In seiner direkten Bedeutung würden wir den unterstrichenen Ausdruck als "j-m einen Gegenstand an den Kopf werfen" verstehen, in diesem Werbetext aber geht es um die umgangssprachliche phraseologische Bedeutung: "j-m etw. Negatives direkt, unverblümt sagen". Beide Ausdrücke verbindet semantisch die Vorstellung von etwas, was einem äußerst unangenehm ist. Der Phraseologismus zeichnet sich dabei durch eine besondere semantische Intensivität aus.

Nur der Charakter der Situation gibt dem Leser die Möglichkeit, die Phraseologismen zu verstehen, die in dem folgenden Werbetext vorkommen:

\section{Lesen macht gesund}

Die Ärztin, Poesietherapeutin und Professorin für Journalistik Silke Heimes schöpft für ihr Buch aus Befragungen von Menschen verschiedener Berufe zu ihren Lektüreerlebnissen und zeigt dabei die vielen Funktionen des Lesens: Bücher können beruhigen und trösten, sie können uns einen Spiegel vorhalten und uns motivieren, Veränderungen in Angriff zu nehmen (Psychologie Heute, April 2018: 84). Beide unterstrichenen Ausdrücke intensivieren die Aussage. Der erste deutet darauf an, dass man die Mängel seines eigenen Charakters oder seines eigenen Verhaltens erkennen soll, um sie zu bekämpfen oder (noch besser) zu beseitigen. Der zweite Ausdruck in Angriff nehmen "mit etwas entschlossen beginnen" hebt den aktiven Charakter der Handlung hervor.

Es sei betont, dass bei der Textproduktion der Faktor des Rezipienten eine überaus wichtige Rolle spielt. Dieser Faktor bedingt die Wahl der Sprachmittel. Abhängig von Alter, Geschlecht, Bildungsniveau, Beschäftigung und Interessen des Adressaten kann derselbe Inhalt auf unterschiedliche Weise modifiziert werden (I. Kitajgorodova, 2015: 41). Aus diesem Grunde bemühen sich die Autoren der Werbetexte darum, nur die idiomatischen Ausdrücke zu benutzen, die nach ihrer Meinung dem jeweiligen Adressaten verständlich sein können, ganz besonders beim Gebrauch von geflügelten Worten, Zitaten aus Werken berühmter Autoren, von Kinderreimen und Kinderspielen sowie Sprichwörtern.

So wird das Biobrot, welches die modernen Leute zur gesunden Ernährung zählen, im Werbetext unter dem Titel Du bist, was du isst! (Brigitte, 2018, Nr. 2: 105) popularisiert. Das Sprichwort fällt durch seine übertragene Bedeutung, Expressivität und seinen scherzhaften Ton auf. 
Der Bibelausdruck Arche Noah ist allgemein bekannt. Aber der Verfasser setzt ihn überraschend in einem Werbetext ein (Gesund leben, 2015, Nr. 2: 90), der verschiedenartige Balkonpflanzen empfiehlt, die den Menschen mit Vitaminen versorgen, und erregt dadurch das Interesse des Rezipienten.

In einer unerwarteten Umgebung werden auch die dem Adressaten seit seinen Schuljahren bekannten Zeilen Kennst du das Land, wo die Zitronen blühn? (Brigitte, 2017, Nr. 9) gebraucht. Es wird nämlich nicht für eine Reise geworben, wie man denken könnte, sondern für ein Buch ("Das kleine Buch der Zitronenpflanzen”). Der Überraschungseffekt kann seine Wirkung auf den Leser nicht verfehlen.

Die Werbung des deutschen Sozialdienstes (Schöner wohnen, Oktober 2018: 198) fordert die Mitbürger auf, an die Kinder zu denken, die ihrer Hilfe bedürfen, und benutzt dabei ein scherzhaftes Lied, das auf den ersten Blick zu dieser Situation gar nicht passt, aber vielleicht gerade dadurch die Aufmerksamkeit des Rezipienten erregt:

Wir haben Hunger, Hunger, Hunger, Wo bleibt das Essen, Essen, Essen,

haben Hunger, Hunger, Hunger,

haben Hunger, Hunger, Hunger,

haben Durst.

bleibt das Essen, Essen, Essen,

bleibt das Essen, Essen, Essen,

Bleibt die Wurst?

\section{Semantische Veränderungen der Phraseologismen im Werbetext}

Von den Werbetextern werden die Phraseologismen Veränderungen verschiedener Art unterworfen, darunter semantischen Veränderungen, die als Resultat des Zusammenwirkens mit dem Text auftreten.

Es kommt vor, dass ein stehender Wortkomplex seinen phraseologischen Charakter verliert und als eine freie Wortverbindung fungiert. So lesen wir im Werbetext Strickplaner (Brigitte, 2019, Nr. 2: 14): Den Faden nicht verlieren: Der Strickplaner sorgt dafür, dass Projekte kein Ideenknäuel bleiben, sondern strukturiert werden - mit Einkaufslisten, Maßen, Inspiration, Fortschritten. Der unterstrichene Phraseologismus ist allen in dem Zusammenhang "den Faden des Gesprächs (nicht) verlieren" in seiner übertragenen Bedeutung bekannt. Hier aber gewinnt er seine direkte Bedeutung zurück und zieht dadurch die Aufmerksamkeit des Lesers auf sich.

In anderen Fällen kommentieren die Autoren einen im Titel oder am Anfang des Textes gebrauchten Phraseologismus in den weiteren Sätzen und lenken dadurch die Aufmerksamkeit des Lesers auf den schon (manchmal nicht deutlich genug) geäußerten Gedanken, wie es im folgenden Werbetext der Fall ist: Schlicht und zeitlos sieht sie aus - doch sie hat es in sich: mit dem neuen Access Gate lassen sich auch Smartphone oder Tablet in die Türkommunikationsanlage von Elcom einbinden. Praktisch, um dem Paketboten aus der Ferne zu sagen, wo er seine Fracht ablegen soll (Schöner wohnen, Juli 2018: 93). Der unterstrichene Phraseologismus klingt nicht ganz deutlich, der Leser versteht nur, dass es sich um eine neue technische Vorrichtung handelt, die dem Verbraucher etwas Besonderes bietet. Die Vorteile von Access Gate werden erst später erklärt.

In einer inhaltlich fremden Umgebung können die phraseologischen Wortkomplexe neue Bedeutungen oder neue Bedeutungsschattierungen bekommen, wie in der Werbung für Kneipp Wirkduschen:

\section{Immer der Nase nach!}

Wie unser Tag startet, können wir morgens unter der Dusche beeinflussen. Denn das neue Aroma-Trio von Kneipp gibt uns ein gutes Gefühl (Brigitte, 2019, Nr. 13: 33). 
Der Titel kann irreführen, denn der Phraseologismus der Nase nach ist dem Rezipienten in der Bedeutung "geradeaus" bekannt und hat scheinbar nichts mit der Dusche gemein, die im Werbetext erwähnt wird. Nur das Wort Aroma gibt dem Leser zu verstehen, dass die Nase hier als Geruchsorgan zu verstehen ist. Das Sem "Richtung” wird durch das Sem "Geruch" ersetzt, was die appellative Funktion des Ausdrucks bedeutend verstärkt.

Der Phraseologismus gut in Form sein gilt in gewöhnlichen Lebenssituationen den Menschen, meistens den Sportlern, die entsprechende körperliche Verfassung erreicht haben, um an einem wichtigen Wettkampf teilzunehmen. Aber in Brigitte Balance Konzept (Brigitte, 2019, Nr. 3: 119) heißt es: Ist dein Leben gut in Form? Der unterstrichene Phraseologismus geht also eine syntaktische Verbindung mit dem abstrakten Substantiv Leben ein, was die Aufmerksamkeit des Lesers aktiviert.

Manche gut bekannte feste Wortkomplexe, die in eine spezifische Umgebung geraten, rufen ein Lächeln des Lesers hervor, wovon die Werbung für heiße Suppen in einer kalten Winterperiode zeugt: Das schönste Warm-up für einen kalten Winter: Suppen und Eintöpfe, die einen glücklich machen! Heiß geliebte Suppen und Eintöpfe (Brigitte, 2019, Nr. 5: 187). Der Adressat versteht den Doppelsinn des Phraseologismus, den der Inhalt des Textes bewirkt. Hei $\beta$ gehört zum Bestand des unterstrichenen Ausdrucks, kann aber auch mit Suppen und Eintöpfen in Verbindung gebracht werden, die man sehr gerne beim kalten Wetter isst. Das Sprachspiel macht den Ausdruck besonders auffällig.

Eine besonders starke Wirkung üben auf den Rezipienten Paradoxe aus, was wir an folgendem Text erleben können: Ihre Nase wird Augen machen! Jetzt neu. Raumdüfte von Kneipp. Kneipp wirkt natürlich. Schon gewusst: Natürliche ätherische Öle beeinflussen aktiv unser Wohlbefinden (Schöner wohnen, Juli 2018: 125). Mit kurzen expressiven Sätzen versucht der Werbetexter die Raumdüfte der Firma Kneipp zu popularisieren, die unser Geruchssinn als angenehm empfindet (im Text wird unser Geruchsorgan die Nase erwähnt). Die erwartete fröhliche Verwunderung des Verbrauchers drückt der Autor mit Hilfe des paradoxen Ausdrucks ihre Nase wird Augen machen aus, der gewöhnlich einem Menschen gilt und nicht einem seiner Organe.

\section{Die Erscheinungsformen von strukturell-semantischen Modifikationen der Phraseologismen}

Die strukturell-semantische Transformation der Phraseologismen, die ein wichtiges Mittel der emotionalen Einwirkung auf den Rezipienten darstellt, entsteht aufgrund der Veränderung der Struktur des Phraseologismus. Als meistverbreitete Erscheinungsform dieser Transformation, die in den untersuchten Werbetexten beobachtet wird, tritt die Erweiterung des festen Wortkomplexes durch Präposition, Postposition oder Interposition auf. Die "Erweiterungswörter" gehen semantische und syntaktische Verbindungen mit den Bestandteilen des Phraseologismus ein, was Veränderungen in der Semantik des festen Wortkomplexes hervorruft und eine bestimmte Spannung schafft, die auf den Adressaten wesentlich einwirkt.

Der Verfasser des weiter zitierten Werbetextes stellt den Unterschied zwischen der ehemaligen und der heutigen Mode auf folgende Weise dar: Einfacher geht Mode nicht - reingeschlüpft, zugeknöpft, gut angezogen! Von ihrem Vorfahren, dem Blaumann, haben diese femininen Teile rein gar nichts mehr (Brigitte, 2019, Nr. 7: 47). Der Ausdruck gar nichts erhielt zwei Erweiterungswörter, die die Einwirkung des Phraseologismus bedeutend verstärken, das eine vorne und das andere - in der Postposition.

In einer anderen Werbung steht in der Postposition ein ganzer Satz, der die Struktur des benutzten Sprichwortes gekürzt wiederholt: Kleider machen Leute, und Fassaden Häuser 
(Schöner wohnen, Mai 2019: 135). So will der Werbetexter den Adressaten überzeugen, dass der Hausbesitzer auf die Fassade seines Hauses ganz besonders achten soll.

Die Erweiterung des Phraseologismus in der Interposition beobachten wir in einem der Sätze der Werbung Zu Besuch bei Marie Montaud: Die Schmuckdesignerin hat ein gutes Händchen für Kleinode: Mitten in Paris verwandelte sie eine ehemalige Fabrik im Hinterhof in ein stilvolles Zuhause (Schöner wohnen, Mai 2019: 14). Eigentlich heißt der Phraseologismus ein Händchen haben "im Umgang mit etwas sehr geschickt sein, das richtige Gefühl für etwas haben". Obwohl der Autor den Ausdruck durch das neutrale Adjektiv gut erweitert, wird die ohnedies emotionsgeladene Bedeutung des Ausdrucks noch etwas verstärkt und somit auch das Interesse des Lesers für Kleinode der erfolgreichen Pariser Schmuckdesignerin Marie Montaud.

Eine Besonderheit der deutschen Sprache ist die Erweiterung der Phraseologismen durch Bildung von Zusammensetzungen: in Form sein > in Topform sein, zum Verkauf anbieten > zum Weiterverkauf anbieten, einen Erfolg landen > einen Überraschungserfolg landen $u$. . Durch diese Art Erweiterung wird die Bedeutung der Redewendungen präzisiert oder mit Emotionen geladen.

Mit großem Effekt wird in Werbetexten auch die Substitution eingesetzt, d. h. der Ersatz einer Komponente des festen Wortkomplexes durch ein funktional ähnliches Wort wie im Titel des Werbetextes Die Kundin ist König (Brigitte, 2016, Nr. 9: 22). Es geht um die Modifikation des bekannten Phraseologismus Der Kunde ist König, der die fürsorgliche Bedienung der Kunden propagiert. Der Ersatz des männlichen Substantivs durch das weibliche klingt wie eine Aufforderung, bei der Beseitigung der technischen Probleme den Kundinnen gegenüber besonders aufmerksam und zuvorkommend zu sein. Der Ersatz verengt die Bedeutung der Aussage, verstärkt aber ihre Emotionalität.

Die Werbetexter greifen auch gern zur Reduktion der Aussage, d. h. zur Auslassung eines Bestandteils oder einiger Bestandteile des Phraseologismus bei der Beibehaltung seiner Kernkomponenten, was dem Wortkomplex eine besondere Ausdruckskraft verleiht. Ein krasses Beispiel dafür ist der Name, den ein Weinbauer einer seiner Weinsorten, einem recht billigen ökologischen Schaumwein gegeben hat: Perlen vor die Säue (Brigitte, 2018, Nr. 9: 18). Der Verbraucher kann diesen Namen als Andeutung darauf verstehen, dass nicht alle Kunden dermaßen kompetent sind, dass sie imstande sind, die Vorteile des angebotenen Produkts verstehen zu können. Die Ironie des Produzenten trägt zum schnellen Absatz des Weines bei. Umso mehr als im Text dieser Werbung ein weiterer modifizierter Phraseologismus steht: Schwein gehabt! Dieser hebt hervor, dass sich der Kunde beim Kauf nicht geirrt hat, der Wein ist dessen wert, gekauft zu werden. In dem letzten Ausdruck liegt auch eine Modifikation vor, sie hat aber grammatischen Charakter und zeigt die Vollendung der Handlung.

Etwas seltener bedienen sich die Verfasser der Werbetexte der Kontamination, wobei sie zwei oder mehr Phraseologismen syntaktisch und semantisch miteinander verbinden wie in der folgenden Werbung für eine kleine Tischlampe: Auf kleinem Fuß, aber mit hellem Köpfchen lebt es sich nicht schlecht (Schöner wohnen, Mai 2016). Auf kleinem Fuß ist ein Gegenteil des Ausdrucks auf großem Fuß leben "aufwendig leben" und klingt bezogen auf einen Menschen ironisch. Heller Kopf (helles Köpfchen) kann einerseits als Bezeichnung einer brennenden Tischlampe aufgefasst werden und andererseits als Lob an die Adresse des Besitzers der Wohnung, in der die Lampe steht. Dem Leser fällt die Zweideutigkeit der genannten Kontamination auf. Sie sollte eigentlich nur der Lampe gelten, kann aber auch auf ihren Besitzer bezogen werden (darauf weist das Verb lebt sich hin), der wahrscheinlich nicht auf den Reichtum hält, aber eine schöne Lampe besitzt. Das Sprachspiel dieses Textes kann seine Wirkung auf den Leser nicht verfehlen. 
Der nächste Werbetext wirkt auf den Leser durch seine Expressivität ein, die infolge der Verletzung der syntaktischen Struktur des Satzes entstanden ist. Es geht um Designerin W. Westwood: Die britische Designerin ist die politischste Modemacherin der Welt-und das macht sie in diesen Zeiten so wichtig wie nie. Grenzen? Kennt ihr Engagement nicht. Und erst recht kein Nein! (Brigitte, 2019, Nr. 2: 30). Der Phraseologismus keine Grenzen kennen verändert seine Struktur: das Substantiv bildet einen Fragesatz. Das Verb und die Negation nicht antworten auf die gestellte Frage. Dabei wird die Negation kein durch die Negation nicht ersetzt, weil sie in der neuen Konstruktion nicht zum Substantiv, sondern zum Verb gehört. Die im Text akkumulierten Veränderungen verleihen ihm eine besondere Ausdruckskraft.

Ein seltenes, aber recht wirksames Mittel der Einwirkung auf den Rezipienten ist die Benutzung der Struktur eines bekannten Wortkomplexes bei der Veränderung seiner lexikalischen Ausfüllung, wie es in der Werbung für eine dem schöpferischen Schaffen gewidmeten Zeitschrift der Fall ist: Auch die großen Genies fangen klein an. Aus Pablo wird Picasso (Brigitte, 2019, Nr. 4: 152). Der unterstrichene Satz hat die Struktur des geflügelten Wortes aus Saulus ein Paulus werden "aus einem Gegner einer Sache zu deren eifrigem Befürworter werden" beibehalten sowie das Kernsem seiner Bedeutung "die Verwandlung einer Person", in unserem Fall ihre schöpferische Entwicklung. Der Name des berühmten Malers ruft beim Leser den Wunsch hervor, die Zeitschrift zu kaufen oder zu abonnieren.

\section{Besonderheiten des Mehrwerts der festen Wortkomplexe mit phraseomatischer Bedeutung}

Hinsichtlich der festen Wortkomplexe mit phraseomatischer Bedeutung kommt die Frage auf, ob ihnen überhaupt der Mehrwert eigen ist. Ihre Bedeutungsstruktur ist nämlich nur etwas komplizierter als die der einfachen Wörter, aber nicht übertragen (A. Kunin,1996: 141). Zahlmäßig bilden sie mit 210 Einheiten eine recht umfangreiche Gruppe. Zum großen Teil sind es Wortkomplexe, die aus einem Substantiv bestehen, das als Bedeutungsträger auftritt, und einem Verb, das bei diesem Substantiv eine formale Funktion erfüllt. Viele Ausdrücke dieser Art kann man durch ein Verb ersetzen: in Kontakt kommen - kontaktieren, zur Ruhe kommen - sich beruhigen, zum Ausdruck bringen - ausdrücken, Notizen machen - notieren, Auftritt haben - auftreten, in Gebrauch sein - gebraucht werden. Andere haben keine verbalen Entsprechungen: in Harmonie leben, Lust haben, Laune machen, Tipps geben, das Leben gestalten, ins Gespräch kommen u. a. m.

Solche Ausdrücke zeichnen sich dadurch aus, dass sie auf die Angehörigkeit des Textes der Schriftsprache und auf den Funktionalstil hinweisen, wovon die unten angegebenen Beispiele zeugen.

Der Verfasser des nächsten Werbetextes empfiehlt seinen Rezipienten die Teilnahme an dem Kongress Kairos, der im April 2018 stattfinden sollte, indem er schreibt: Dieser Kongress ist eine besondere Zeitqualität voller Möglichkeiten, in der Menschen die Gelegenheit haben, das Leben zu gestalten (Psychologie Heute, 2018, Nr. 4: 105). Beide unterstrichenen festen Wortkomplexe gehören zur Schriftsprache und werden oft in der Presse eingesetzt.

In Werbetexten verschiedener Zeitschriften finden wir Sätze wie: Spitziges Gelb, saftiges Orange und Limonegrün haben im Sommer ihren Auftritt; Alltagsgegenstände sind ständig $\underline{\text { in }}$ Gebrauch; Psychologen, Ärzte, Ernährungsberater können über ihre Zusatzfunktion mit ihren Patienten in Kontakt treten. In allen unterstrichenen Ausdrücken tragen die Substantive starke Betonung, wodurch die Intention des Autors hervorgehoben wird. Der Ersatz der Redewendung durch das synonymische Verb schwächt die Betonung ab und verwischt den Sinn der Aussage. 


\section{Schlussfolgerungen und Ausblick}

Aus dem Gesagten lässt sich Folgendes schließen:

Die Aufgabe der Werbung besteht darin, durch relativ kurze Texte dem Empfänger die angebotenen Produkte möglichst überzeugend zu präsentieren, um ihn zur Handlung zu provozieren. Als Mittel, den potentiellen Kunden auf diese Produkte aufmerksam zu machen, eignen sich wohl am besten die festen Wortkomplexe, die sich durch ihre semantische Auffälligkeit auszeichnen.

Die Untersuchung hat gezeigt, dass allen Phraseologismen des Forschungskorps der semantische Mehrwert eigen ist, der sie konnotativ von ihren nichtphraseologischen Entsprechungen unterscheidet und in den einzelnen ausgesonderten Gruppen unterschiedlich zum Ausdruck kommt.

Besonders auffällig sind die festen Wortkomplexe mit idiomatischer Bedeutung. Ihren semantischen Mehrwert kann die übertragene Bedeutung bedeutend verstärken, die nicht selten vom Charakter der vom Autor geschaffenen Situation abhängig ist.

Eine überaus wichtige Erscheinungsform des semantischen Mehrwerts in Werbetexten ist der Einsatz von Sprichwörtern, geflügelten Worten, Zitaten aus bekannten Werken, Kinderspielen, Kinderreimen u. Ä. Sie gehören zum sprachfixierten Erfahrungsschatz der Sprachgemeinschaft und fallen durch ihre übertragene Bedeutung, ihre Expressivität und Intensivität sowie durch ihren scherzhaften Ton auf.

Eine überaus wichtige Rolle bei der Schaffung des semantischen Mehrwerts spielt die Modifikation des Phraseologismus, die in Erweiterung oder Reduktion des Ausdrucks, Substitution, Kontamination oder Verletzung der grammatischen Struktur des Phraseologismus besteht.

Dem Adressaten fallen auch der Gebrauch von Phraseologismen in ihrer freien Bedeutung, ihre Realisierung in einer scheinbar unpassenden Umgebung, das Kommentieren der im Titel gebrauchten stehenden Redewendung im Text auf.

Die Untersuchung von Wendungen mit phraseomatischer Bedeutung zeigt, dass zu ihrer phraseologischen Bedeutung auch ein semantischer Mehrwert gehört, der aber anders zum Ausdruck kommt. Zu den Besonderheiten dieser Ausdrücke gehören ihre deutlich ausgeprägte funktional-stilistische Angehörigkeit und ihre Fähigkeit, das sinnwichtige Wort mit Hilfe der Betonung hervorzuheben. Solcher Art Wortkomplexe können nur mit Hilfe der Erweiterung des Ausdrucks modifiziert werden, was die Aussage deutlich intensivieren kann.

Wir halten es für wichtig, bei den weiteren Recherchen stilistische Besonderheiten der Phraseologismen mit idiomatischer und fraseoidiomatischer Bedeutung zu untersuchen.

Da die festen Wortkomplexe ihre volle kommunikative Wirkung durch die Einbettung in den Text erzielen, wo sie bestimmte Funktionen übernehmen und zum Ausbau des Textes beitragen, scheint es uns auch wichtig, die textbildenden Potenzen der Phraseologismen in Werbetexten zu erforschen.

\section{Referenz}

Avksentiev, L. H. (1988). Suchasna ukrainska mova. Frazeolohiia [Modern Ukrainian language. Phraseology]. Kharkiv: Vydavnytstvo pry Kharkivskomu derzhavnomu universyteti. [in Ukrainian].

Baran, Y. A., Zymomria, M. I., Bilous, O. M., Zymomria, I. M. (2008). Frazeolohiia: znakovi velychyny. Navchalnyi posibnyk dlia studentiv fakultetiv inozemnykh mov [Phraseology: sign values. Training manual for foreign department students ]. Vinnytsia: Nova knyha. [in Ukrainian] 
Burger, H. (2010). Phraseologie. Eine Einführung am Beispiel des Deutschen [Phraseology. Introduction through the example of the German language]. Bielefeld: Erich Schmidt Verlag [in German]

Drumm, D. (2004). Semantischer Mehrwert und Multifunktionalität von Phraseologismen in der englischsprachigen Anzeigenwerbung. Wissenschaftliche Arbeit zur Erlangung des Doktorgrades [Semantic added value and multifunctionality of phraseologisms in English-language advertising. Research work for PhD degree]. Trier: Universität Trier. [in German]

Fleischer, W. (1982). Phraseologie der deutschen Gegenwartssprache [Phraseology of the modern English language]. Leipzig: VEB Bibliographisches Institut. [in German]

Kitajgorodova, I. N. (2015). Lingvisticheskij analiz teksta. Uchebnoe posobie [Linguistic analysis of text. Training manual]. Astrahan: Izdatelskij dom “Astrahanskij universitet”. [in Russian] Kühn, P, (1994). Pragmatische Phraseologie: Konsequenzen für Phraseologie und Phraseodidaktik. [Pragmatic phaseology: consequences for phraseology and phraseodidactics]. In: B. Sandig. Europhras 92. Tendenzen der Phraseologieforschung. Studien zur Phraseologie und Parömiologie. Bochum:Brockmeyer. [in German]

Kunin, A. V. (1996). Kurs frazeologii sovremennogo anglijskogo jazyka [The Course of Phrazeology of the Modern English Language]. Moscow: Vysshiya shkola. [in Russian]

Selivanova, O. O. (2010). Linhvistychna entsyklopediia [Linguistic Encyclopaedia]. Poltava: Dovkillia. [in Ukrainian].

Shahovskij, V. I. (2008). Kategorizaciya emocij leksiko-semanticheskoj sistemy yazyka [The categorization of emotions of lexical-semantic language system]. Moscow: Izdatelstvo LKI. [in Russian]

Uzhchenko, V. D. (1988). Narodzhennia $i$ zhyttia frazeolohismu [The birth and the life of phraseologism]. Kyiv: Radianska shkola. [in Ukrainian] 